Original paper

\title{
Prediction methods for synchronization of scanned ion beam tracking
}

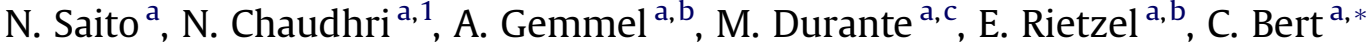 \\ ${ }^{a}$ GSI Helmholtz Centre for Heavy Ion Research GmbH, Planckstraße 1, 64291 Darmstadt, Germany \\ ${ }^{\mathrm{b}}$ Siemens AG, Healthcare Sector, Imaging \&' Therapy, Particle Therapy, Hofmannstr, 26, 91052 Erlangen, Germany \\ ${ }^{\mathrm{c}}$ Technical University of Darmstadt, Department of Condensed Matter Physics, 64289 Darmstadt, Germany
}

\section{A R T I C L E I N F O}

Article history:

Received 29 March 2012

Received in revised form

5 August 2012

Accepted 11 August 2012

Available online 11 September 2012

\section{Keywords:}

Scanned ion beam

Radiotherapy

Organ motion

Beam tracking

\begin{abstract}
A B S T R A C T
Beam tracking as a mitigation technique for treatment of intra-fractionally moving organs requires prediction to overcome latencies in the adaptation process. We implemented and experimentally tested a prediction method for scanned carbon beam tracking. Beam tracking parameters, i.e. the shift of the Bragg peak position in 3D, are determined prior to treatment in 4D treatment planning and applied during treatment delivery in dependence on the motion state of the target as well as on the scanning spot in the target. Hence, prediction is required for the organ motion trajectory as well as the scanning progress to achieve maximal performance. Prediction algorithms to determine beam displacements that overcome these latencies were implemented. Prediction times of $25 \mathrm{~ms}$ for target spot prediction were required for $\sim 6 \mathrm{~mm}$ water-equivalent longitudinal beam shifts. The experimental tests proved feasibility of the implemented prediction algorithm.
\end{abstract}

๑ 2012 Associazione Italiana di Fisica Medica. Published by Elsevier Ltd. All rights reserved.

\section{Introduction}

Target volume conformal dose delivery is one of the major concerns in radiotherapy. Due to advantageous physical properties, scanned ion beams $[1,2]$ allow excellent target conformation for stationary tumours $[3,4]$. The main current detriment of ion beam scanning is its sensitivity to organ motion due to interplay effects between scanned beam and moving tumour [5-7]. One possibility to overcome the sensitivity to target motion is beam tracking that was originally proposed for photon beam therapy [8] and is technically available for several treatment techniques [9-11]. Scanned ion beam tracking requires adaptation of the pencil beam during irradiation. The adaptation is relative to the reference treatment plan that is optimized on a stationary reference phase of the underlying time-resolved computed tomography (4DCT) (e.g. endexhale). Optimization results in treatment plans that specify each beam spot by its energy, beam width, beam position $(x, y)$, beam intensity level and the number of particles deposited to each scanning spot (raster point $r$ ). Adaptation of the beam parameters during delivery is based on the motion state of the target (motion phase $m$ ), the currently irradiated raster point $r$, and dedicated 4D treatment planning that provides a look-up table (LUT) of beam

\footnotetext{
* Corresponding author. Tel.: +496159 712197.

E-mail address: c.bert@gsi.de (C. Bert).

1 NC meanwhile works for Heidelberg Ion Beam Therapy Center (HIT).
}

adaptation parameters (lateral beam displacements $d_{x}$ and $d_{y}$ via the scanner magnets, and range change $d_{z}$ via a range shifter, details in [12]) for all combinations of raster points $r$ and motion phases $m$. Especially the particle range adaptation $d_{z}$ can vary even for neighbouring beam positions depending on the tissue composition in the entrance channel [11].

Between detecting target motion state $m$ as well as raster point $r$ and beam adaptation on target there are time delays of the processes in the beam tracking system. We determined a delay and response of $<1 \mathrm{~ms}$ for the lateral beam tracking process and a communication delay of $11 \mathrm{~ms}$ to the range shifter plus a range shift dependent response time for range adaptation (e.g. $16 \mathrm{~ms}$ response time for $5 \mathrm{~mm}$ water-equivalent (WE) range shift) [12]. In comparison to multi-leaf collimator based tracking with typical response times of more than $100 \mathrm{~ms}$ [13] our system response is very fast but since the range adaptation response time is comparable to the irradiation time per raster point ( $\sim 10 \mathrm{~ms}$ at GSI Helmholtz Centre for Heavy Ion Research (GSI)) we have to incorporate prediction methods to obtain maximal precision in range adaptation. A potential solution for range adaptation with negligible delays is based on an ion optical method rather than mechanical drives. Even though the method has been implemented for performance studies at GSI [17] full clinical implementation requires change of beam line magnets that is not easily performed at existing therapy facilities. A short-term solution that would drastically reduce the response time of range adaptation is thus not in sight. 
For lateral compensation currently no prediction is required. In this work we included also parameters for lateral prediction that could become important, e.g. if irradiation times per raster point decrease in future implementations. To overcome the system latency of the range adaptation we require not only motion prediction to determine the motion phase $m$ at the time of the (delayed) adaptation as in the other tracking modalities (review on motion prediction by [14]). We also need to predict the raster point $r$ to fully synchronize beam tracking with target motion.

This prediction feature has been implemented in the beam tracking system at GSI (12), however a detailed description of the prediction methods has never be reported. In the scope of this work the implemented prediction algorithms and the experimental verification of the prediction method for scanned ion beam tracking are described. The experiments focused on raster point prediction. In an artificial setup, distinct changes of a pattern irradiated on radiographic films allow judgement of the suitability of the raster point prediction parameters.

\section{Materials and methods}

\section{Prediction method}

Algorithms to predict beam adaptation parameters were implemented in the beam tracking unit (BTU) of the therapy control system (TCS) in order to determine adaptation parameters that include the time delay of the tracking process. Different prediction algorithms are required for motion phase prediction (prediction time $t_{m}$ ) and raster point prediction (prediction time $t_{r}$ ). In addition, separate prediction times are supported for lateral prediction (upper suffix: lat.) and range adaptation (upper suffix: range) since the corresponding tracking sub-systems show different time responses. This results in four prediction: $t_{m}^{\text {lat }}, t_{r}^{\text {lat }}, t_{m}^{\text {range }}$, and $t_{r}^{\text {range }}$.

For motion prediction due to the fast response of our system we currently use linear extrapolation of the motion signal measured with our laser triangulation distance sensor (sampling rate $\sim 1 \mathrm{kHz}$ ) [12] rather than the state of the art motion prediction reported in the literature [14]. The BTU operates in a calculation loop ( $0.5 \mathrm{~ms} / \mathrm{loop})$ that reads motion signal and raster point number, determines the beam adaptation parameters, and distributes them to lateral as well as range modulation systems. To be less sensitive to fluctuations in the motion signal we continuously average the motion signal with the 5-10 prior data points at BTU.
For the linear extrapolation, we use two averaged data points which have a certain temporal spacing $t_{i}$ to each other to determine the slope $\varphi$ of the linear extrapolation (see Fig. 1). We typically use $t_{i}=15 \mathrm{~ms}$ as a compromise of waiting long enough to avoid extrapolations of microscopic fluctuation and a reasonably short spacing to minimize deviations related to the only linear approximation. Future motion signals are extrapolated for the prediction times $t_{m}^{\text {range }}$. Each predicted motion signal is then used to determine the corresponding motion phases as the predicted motion phases $t_{p}^{\text {lat }}$ and $t_{p}^{\text {range }}$. Motion phase determination can be phase-based or amplitude-based and typically the motion phase represents one state of the 4DCT used for treatment planning. Example data for the motion prediction process are illustrated in Fig. 1. Based on two mean values that are spaced by at least $t_{i}$ the slope of the linear extrapolation $\varphi$ is calculated and used in the following $t_{i}$ milliseconds to predict the range motion signal from the motion amplitude offset $a_{m}^{\text {range }}$ at a prediction time $t_{m}^{\text {range }}$. The same steps are performed for prediction in the lateral direction with $a_{m}^{\text {lat }}$ and $t_{m}^{\text {lat }}$. Based on the predicted motion signals the predicted motion phases for lateral $m_{m}^{\text {lat }}$ and range adaptation $m_{m}^{\text {range }}$ are determined (right axis scale in the panel (c) in Fig. 1). In this example we used 31 motion phases with amplitude based motion phase determination.

In order to predict the irradiation time of future raster points, progress of the irradiation has to be known. The irradiation time of a raster point $r$ can be determined by the beam delivery rate (particle extraction from the accelerator) and the optimized number of particles per raster point. At GSI the extraction does not yet result in a constant rate but fluctuates within a beam pulse [15] and thus does not allow to estimate the irradiation time of raster points with an accuracy of milliseconds prior irradiation. For a specific treatment plan the fluctuations are comparable within short time periods since the accelerator conditions do not change. Instead of estimating the irradiation time based on nominal data we thus implemented an estimation method based on measured data for a specific treatment plan. Just prior the planned irradiation a test irradiation is performed to determine the duration of irradiation for each raster point as a relative irradiation time. These data are stored in a so-called beam sequence file that is then used in the actual experiment. Example data of relative irradiation times for four different runs of a specific plan are shown in Fig. 2a. The example data were obtained at GSI sequentially in the same day. As it was expected, relative irradiation times do not change significantly from one run to another. For one of these runs the accumulated
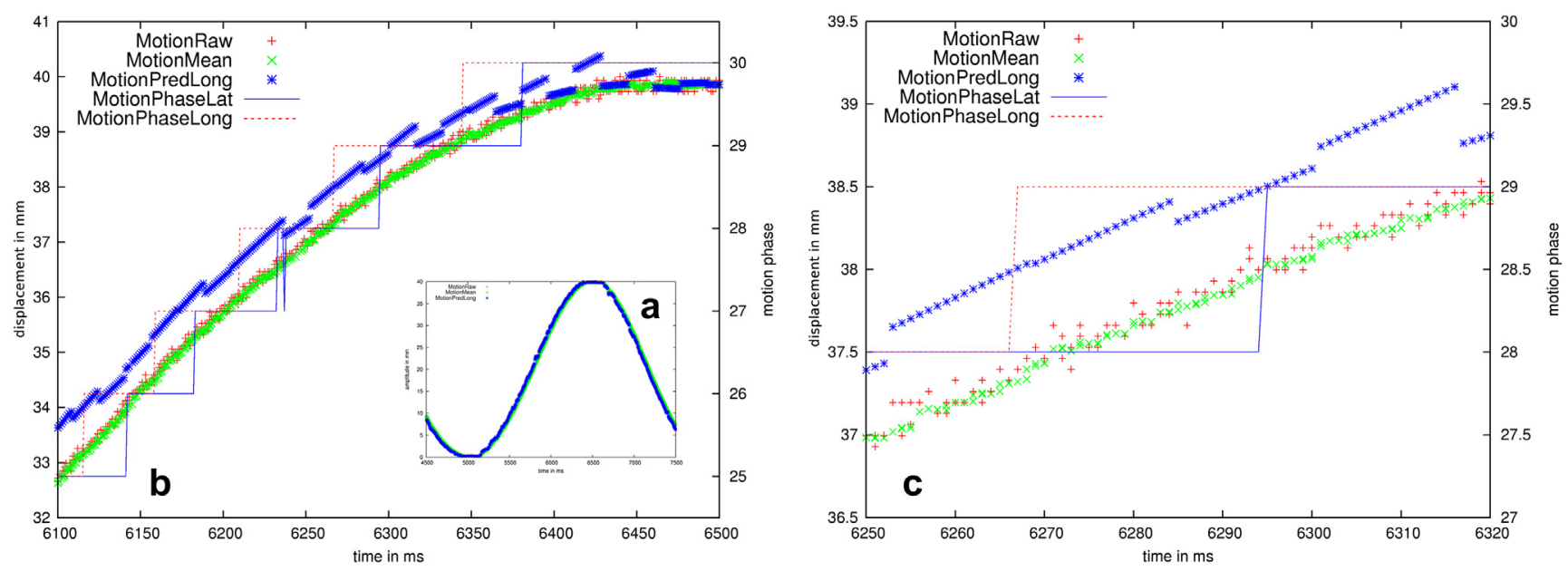

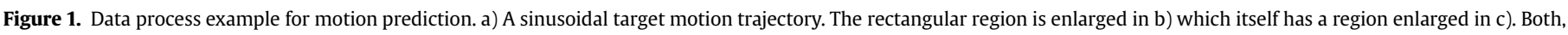
b) and c) show the motion signal raw data $(+)$ as determined by the laser sensor. 5-10 raw data points (interval $500 \mu$ s) contribute to a mean motion signal $(\times)$. 


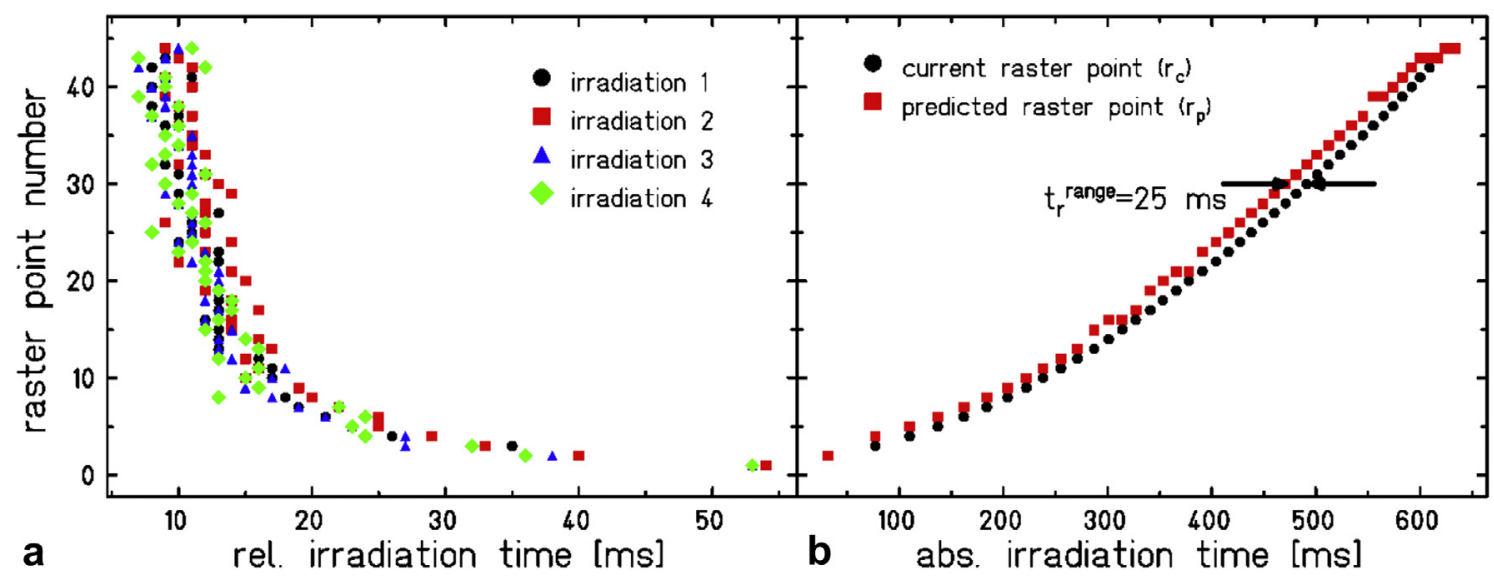

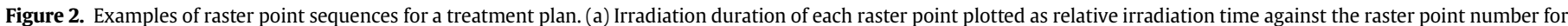

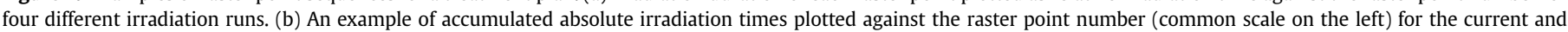
predicted raster point numbers, $r_{c}$ and $r_{p}$, respectively for a raster point prediction time $t_{r}^{\text {range }}$ of $25 \mathrm{~ms}$.

irradiation time (i.e. absolute irradiation time) is shown against the raster point number $r$ in Fig. $2 b$ to demonstrate the temporal progress of raster point number for this example plan. For the prediction of raster points the BTU compares the prediction time $t_{r}^{\text {range }}\left(t_{r}^{\text {lat }}\right.$ for lateral) with the pre-measured irradiation times of the following raster points stored in the beam sequence file. The BTU can thus determine which raster point will be irradiated after the specified delay $t_{r}^{\text {range }}$ ( $t_{r}^{\text {lat }}$ for lateral). We refer to this prediction raster point as $r_{p}$. Figure $2 \mathrm{~b}$ also shows an example of predicted raster points $r_{p}$ for $t_{r}^{\text {range }}=25 \mathrm{~ms}$.

According to $m_{p}$ and $r_{p}$ the predicted beam displacements $d_{m_{p}, r_{p}}=\left(d_{x}, d_{y}, d_{z}\right)_{m_{p}, r_{p}}$ can be selected from the LUT and sent as command values to the tracking subsystems. Figure 3 illustrates the described two predictions for motion and raster point to select suitable beam displacements from the pre-calculated LUT.

\section{Experimental setup}

Measurements to verify the prediction method were performed with the scanned carbon beam tracking at GSI. We used an irradiation plan and a setup that was designed for assessment of the implemented prediction method, i.e. some of the irradiation parameters are not typical for patient treatment plans. The irradiation plan consisted of a single iso-energy slice $(400 \mathrm{MeV} / \mathrm{u})$ with $5 \times 9$ raster points in a $6 \mathrm{~mm}$ grid as a rectangular shaped target.

\section{motion phase $\boldsymbol{m}$}

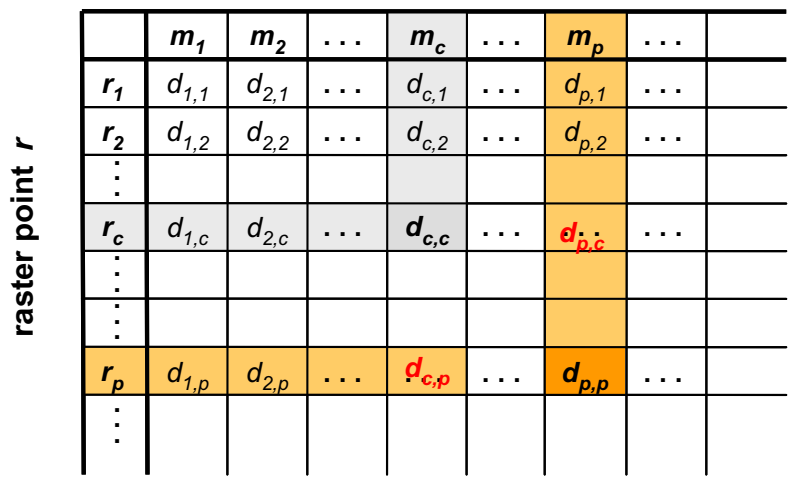

Figure 3. A schematically illustrated LUT. The current motion phase $m_{c}$, current raste number $r_{c}$, the predicted motion phase $m_{p}$, and the predicted raster number $r_{p}$ can be calculated, and the predicted beam displacements $d_{p, p}$ can be sent as command values.
We chose a small beam size of $2.2 \mathrm{~mm}$ full width at half maximum (FWHM) to minimize the overlap of the individual beam positions in contrast to the preferred overlaps in patient treatments. For the assessment of the prediction effect the small overlap allows us to clearly see the irradiation of individual raster points. The raster points were irradiated with a left-right scanning direction starting from the bottom-left point (last point at top-right). The time duration for each beam spot was measured (14.6 $\mathrm{ms}$ as an average value) and stored as a beam sequence file on the beam tracking unit (see Fig. 2a). Total irradiation time was thus $\sim 650 \mathrm{~ms}$.

The experimental set-up is schematically shown in Fig. 4. As detector we used a radiographic film positioned on a sliding table distal of a polymethylmethacrylate (PMMA) absorber in the Bragg peak region of the $400 \mathrm{MeV} / \mathrm{u}$ carbon ion beam. The table moved left-right in beam's eye view (BEV) with an average target motion speed of $13 \mathrm{~mm} / \mathrm{s}$; the motion started synchronized to the irradiation of the first beam position. The average speed of $13 \mathrm{~mm} / \mathrm{s}$ is slightly below the maximum speed of a modelled speed of respiratory motion $(17 \mathrm{~mm} / \mathrm{s})[12,16]$. Due to table motion lateral beam tracking is required to produce the planned response on the film, i.e. to hit the film at all the $5 \times 9$ raster point positions. To introduce also range changes we used two different PMMA absorber thicknesses and positioned the absorber stationary proximal to the sliding table: In BEV right of the isocenter the absorber was adjusted $5 \mathrm{~mm}$ (10 $\mathrm{mm}$ ) thicker than in BEV left (16 cm thickness) (see Fig. 4). The additional PMMA of $5 \mathrm{~mm}$ (10 mm) corresponds to $\sim 6 \mathrm{~mm}(\sim 12 \mathrm{~mm})$ WE thickness. With this absorber arrangement the ion beam stops on the film in BEV left of the isocenter but on the right side the beam does not reach the film without temporally synchronized beam range tracking. If the range is not adjusted in time this will result in an empty grid position on the film that will be clearly visible in the blackening distribution due to the reduced overlap in the $6 \mathrm{~mm}$ grid (Fig. 4ab). With appropriate range tracking that includes prediction to overcome delays and response times also beam positions in BEV right of the isocenter will result in the planned film response (Fig. 4cd).

\section{Experimental procedure}

Two irradiations with a stationary setup were performed without beam tracking to confirm the set-up and shape of the irradiation pattern. In the first experiment, the treatment plan was delivered without range adaptation (all beam positions at a beam energy of $400 \mathrm{MeV} / \mathrm{u}$ ), expecting that only beam positions in $\mathrm{BEV}$ 


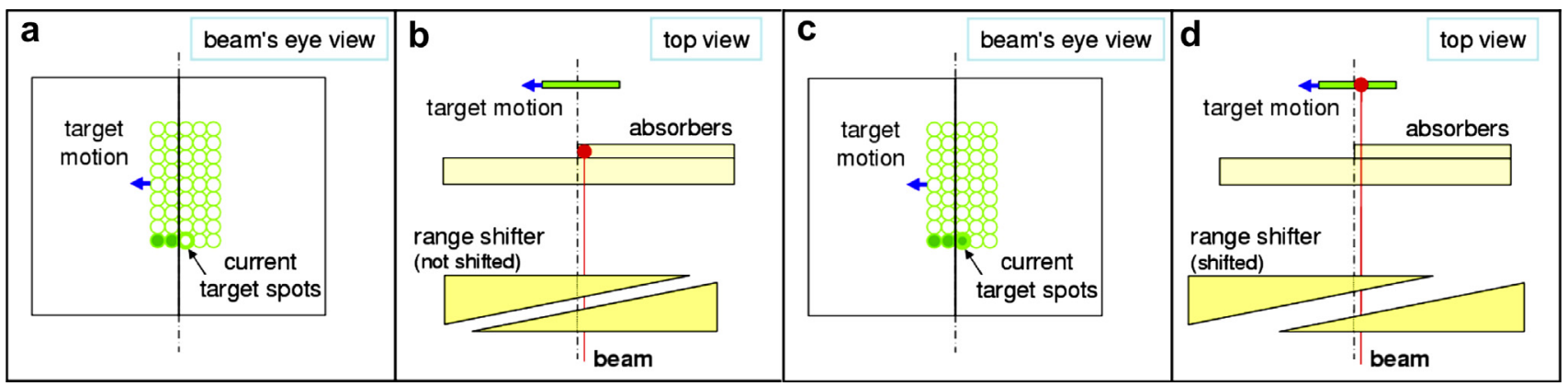

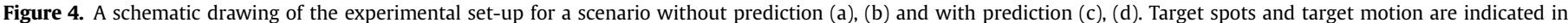

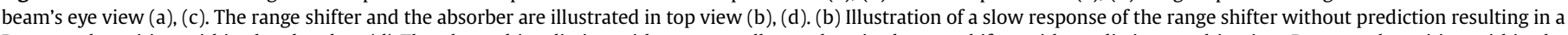

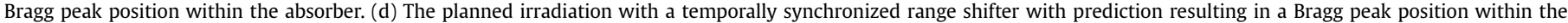
target area.

left of the isocenter will cause blackening on the film. In the second irradiation, beam penetration through the thicker absorber in BEV right was confirmed by requesting the corresponding higher beam energy from the accelerator $(406 \mathrm{MeV} / \mathrm{u}$ for $5 \mathrm{~mm}$ PMMA, $411 \mathrm{MeV} / \mathrm{u}$ for $10 \mathrm{~mm}$ PMMA) after the irradiation of the BEV left energy layer (requiring $5 \mathrm{~s}$ for the energy change) to confirm the set-up and film blackening in the whole target area.

For the case of beam tracking with a moving target we expected from our measurements in the accuracy study [12] an influence of delays and response times in the range domain but not laterally. For a $5 \mathrm{~mm}$ PMMA (10 mm PMMA) equivalent range shift the expected prediction times were $20-30 \mathrm{~ms}$. We thus performed experiments with raster point prediction times $t_{r}^{\text {range }}$ of 20,25 , and $30 \mathrm{~ms}$ for $5 \mathrm{~mm}$ PMMA, and 25, 30, and $35 \mathrm{~ms}$ for $10 \mathrm{~mm}$ PMMA thickness. The motion prediction parameter $t_{m}^{\text {range }}$ was kept constant at $25 \mathrm{~ms}$ to demonstrate the clear difference of the raster point prediction effect in this report. In all experiments lateral prediction was not necessary to apply $\left(t_{m}^{\text {lat }}=t_{r}^{\text {lat }}=0\right)$ because the expected delay of about $1 \mathrm{~ms}$ was considerably less than the time duration of a motion phase and a raster point.

\section{Results}

Figure 5 shows the radiographic film response for the measurements with and without beam tracking together with the planned location of target spots shown as circles. Figure 5A shows film images without beam tracking on static films. As it was planned, the beam of $400 \mathrm{MeV} / \mathrm{u}$ reached to the film for the left two columns of the target (thin absorber) but was stopped in the additional absorber material in the right three columns (Fig. 5Aa). For irradiations with two beam energies, one for the left two columns and the other for the right three columns, the beams reached to the film for the whole target area (Fig. 5Ab) and thus provide a positive control of the experimental procedure.

Figure $5 \mathrm{~B}$ shows the resulting film response distributions for beam tracking with the $5 \mathrm{~mm}$ PMMA absorber. Figure 5Ba shows the result with motion prediction but without raster point prediction $\left(t_{r}^{\text {range }}=0\right)$. The blackening distribution shows white (or light grey) spots on the film when the scanned beam moved to BEV right where the additional absorber was covering the target spots. Since the film was moving with $13 \mathrm{~mm} / \mathrm{s}$ during the measurements the transition to the thicker absorber was shifted relatively to the film by roughly one beam spot (spacing: $6 \mathrm{~mm}$ ) to the right towards the end of the irradiation (irradiation time: $650 \mathrm{~ms}$, details: Fig. 5Ba top row). The results with different raster point prediction parameters $t_{r}^{\text {range }}$ are also shown in Fig. 5B. Raster point prediction with $t_{r}^{\text {range }}=20 \mathrm{~ms}$ resulted in a pattern with white spots (Fig. $5 \mathrm{Bb}$ ) similar to the results without raster point prediction (Fig. 5Ba). With $t_{r}^{\text {range }}=25 \mathrm{~ms}$ (Fig. 5Bc) nearly all of the target spots were filled black comparable to the stationary reference in Fig. $5 \mathrm{Ab}$ and with a clear improvement in comparison to the previous case with $t_{r}^{\text {range }}=20 \mathrm{~ms}$. The irradiation pattern with $t_{r}^{\text {range }}=30 \mathrm{~ms}$ shows white spots on the film when the scanning direction was right-to-left (Fig. 5Bd, target spot in the 2nd row from top). In this case the adaptation to reduce range was too early and shifted the beam into the absorber. We did not show the results for

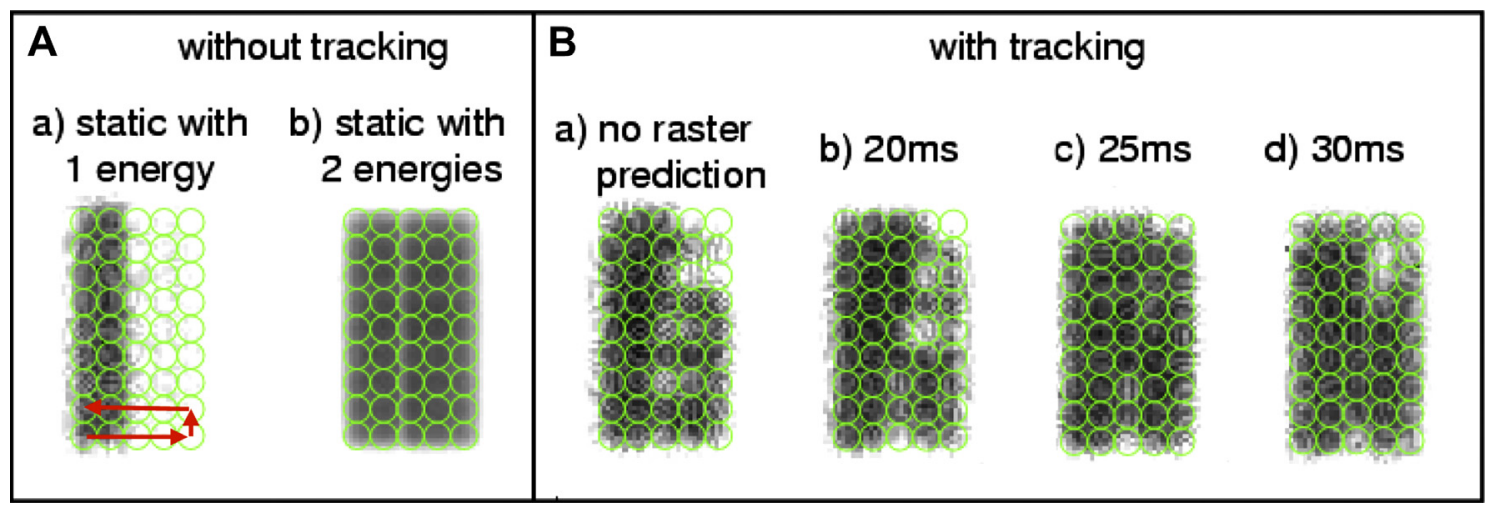

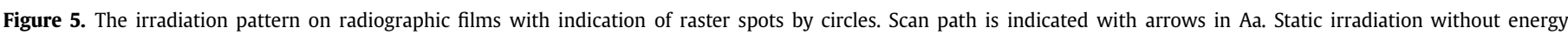

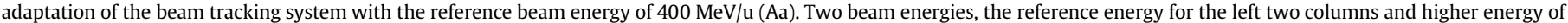

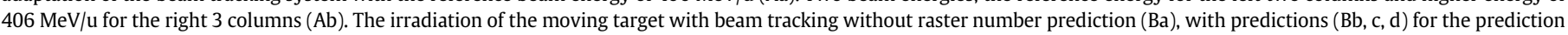
parameter $t_{r}=20,25,30 \mathrm{~ms}$, respectively. 
$t_{r}^{\text {range }}<20 \mathrm{~ms}$ and $t_{r}^{\text {range }}<30 \mathrm{~ms}$ because they led to worse results than the ones shown in Fig. 5B as it is expected from the estimated prediction time. Thus the result with $t_{r}^{\text {range }}=25 \mathrm{~ms}$ was optimal among the tested irradiations.

For the measurements with $10 \mathrm{~mm}$ PMMA thickness the results were qualitatively comparable. The optimal $t_{r}^{\text {range }}$ was determined at $30 \mathrm{~ms}$.

\section{Discussion}

The 3D beam tracking was performed with or without raster point predictions for the longitudinal beam tracking to demonstrate that raster point prediction which is unique to the scanned ion beam therapy with the existing beam tracking system at GSI offers the possibility to overcome the latency effect of longitudinal beam tracking. For the demonstration, a fast range shift of $5 \mathrm{~mm}$ (or $10 \mathrm{~mm}$ ) PMMA thickness corresponding to $\sim 6 \mathrm{~mm}$ (or $12 \mathrm{~mm}$ ) in water was required frequently (1 shift per 4-6 scanning spots). The results for the measurements with/without raster point prediction show clearly that a synchronized irradiation was achieved by applying the raster point prediction of $25 \mathrm{~ms}$ for $5 \mathrm{~mm}$ PMMA (30 ms for $10 \mathrm{~mm}$ PMMA) with a target motion prediction of $25 \mathrm{~ms}$. The raster point prediction parameters of $20 \mathrm{~ms}$ (30 ms) for $5 \mathrm{~mm}$ PMMA and $25 \mathrm{~ms}$ (35 ms) for $10 \mathrm{~mm}$ PMMA were too short (long) to perform a synchronized irradiation in this study.

For the measurements in this work the scanning duration for each spots was observed to be $14.6 \mathrm{~ms}$ as an average with a minimum of $8 \mathrm{~ms}$ and a maximum of $53 \mathrm{~ms}$. Due to intensity structure of the used ion beams from the synchrotron accelerator irradiation duration of a spot was longest in the beginning of the irradiation and after roughly 10 spots the duration is rather constant with approximately $10 \mathrm{~ms}$ (see Fig. 2a). As it was presented in the Fig. 5Bc the irradiation with $25 \mathrm{~ms}$ raster point prediction shows good results for such beam scanning speeds ( $\sim 10 \mathrm{~ms} / \mathrm{spot}$ ) which are comparable to the typical scanning progress used for patient treatments at GSI.

Impact of raster point prediction was demonstrated in this study for a specific experimental case, and the technical functionality was clearly verified. The experimental parameters were chosen such that the range change amplitudes as well as timing as the critical parameters are comparable to the ones determined in a treatment planning study for beam tracking for lung cancer treatment. Therefore the demonstrated prediction functionality can be considered to be beneficial for clinical cases. The main difference is that the range change pattern is very irregular with respect to direction and amplitude and can be more frequent, i.e. from raster point to raster point. Hence for patient treatments that most likely show a large variation of range shift amplitude a constant raster point prediction time $t_{r}^{\text {range }}$ would not be the best choice. For these cases we propose to use prediction times that are dependent on the required range change, i.e. $t_{r}^{\text {range }}=t_{r}^{\text {range }}\left(d_{z}\right)$. In order to investigate prediction parameters for patient treatment plans we plan $4 \mathrm{D}$ treatment planning studies based on the data obtained in this study. These treatment planning studies should also address the permissible error in the prediction time as this might influence the dose deposition as shown in Fig. 5. We expect that the dosimetric influence of the prediction error depends mainly on the range shift amount and the duration of the irradiation of a raster point. Both parameters were fixed in the performed experiments but will frequently change in a real patient.

\section{Conclusion}

A method to predict beam displacement parameters for a scanned ion beam tracking of moving targets was implemented in the beam tracking system at GSI. The method utilizes not only target motion prediction but also prediction of the future irradiation spot (raster point prediction) to determine suitable beam displacement commands in time. The experimental test resulted in a synchronized irradiation with $25 \mathrm{~ms}(30 \mathrm{~ms})$ raster point prediction times for range shifts of $\sim 6 \mathrm{~mm}(\sim 12 \mathrm{~mm})$ waterequivalent.

\section{Conflict of interest statement}

This work was in part supported by Siemens AG, Healthcare Sector, Imaging \& Therapy, Particle Therapy. Two authors, ER and AG, are employed by Siemens AG and guest researchers at GSI.

\section{Acknowledgements}

The authors appreciate Prof. Dr. Dr. h.c. G. Kraft supporting the present work and encouraging us continuously. The authors thank our colleagues in GSI and the Heidelberg Ion Therapy facility for their technical supports. The present work is partially funded by Siemens AG, Healthcare Sector, Workflow and Solutions, Particle Therapy.

\section{References}

[1] Haberer T, Becher W, Schardt D, Kraft G. Magnetic scanning system for heavy ion therapy. Nucl Instrum Meth A 1993;330:296-305.

[2] Pedroni E, Bacher R, Blattmann H, Bohringer T, Coray A, Lomax A, et al. The 200-MeV proton therapy project at the Paul Scherrer institute: conceptual design and practical realization. Med Phys 1995;22:37-53.

[3] Wilson RR. Radiological use of fast protons. Radiology 1946;47:487-91.

[4] Kraft G. Tumor therapy with heavy charged particles. Prog Part Nucl Phys 2000; $45:$ s473-544.

[5] Phillips MH, Pedroni E, Blattmann H, Boehringer T, Coray A, Scheib S. Effects of respiratory motion on dose uniformity with a charged particle scanning method. Phys Med Biol 1992;37:223-33.

[6] Seiler PG, Blattmann H, Kirsch S, Muench RK, Schilling C. A novel tracking technique for the continuous precise measurement of tumour positions in conformal radiotherapy. Phys Med Biol 2000;45:103-10.

[7] Bert C, Grözinger SO, Rietzel E. Quantification of interplay effects of scanned particle beams and moving targets. Phys Med Biol 2008;53:2253-65.

[8] Keall PJ, Kini VR, Vedam SS, Mohan R. Motion adaptive X-ray therapy: a feasibility study. Phys Med Biol 2001;46:1-10.

[9] Schweikard A, Glosser G, Bodduluri M, Murphy MJ, Adler JR. Robotic motion compensation for respiratory movement during radiosurgery. Comput Aided Surg 2000;5:263-77.

[10] D'Souza W, Naqvi SA, Yu CX. Real-time intra-fraction-motion tracking using the treatment couch: a feasibility study. Phys Med Biol 2005;50:4021-33.

[11] Bert C, Saito N, Schmidt A, Chaudhri N, Schardt D, Rietzel E. Target motion tracking with a scanned particle beam. Med Phys 2007;34:4768-71.

[12] Saito N, Bert C, Chaudhri N, Gemmel A, Schardt D, Rietzel E. Speed and accuracy of a beam tracking system for treatment of moving targets with scanned ion beams. Phys Med Biol 2009;54:4849-62.

[13] Murphy MJ, Dieterich S. Comparative performance of linear and nonlinear neural networks to predict irregular breathing. Phys Med Biol 2006;51:5903-14.

[14] Murphy MJ. Tracking moving organs in real time. Semin Radiat Oncol 2004; 14:91-100.

[15] Grözinger SO, Rietzel E, Li Q Bert C, Haberer T, Kraft G. Simulations to design an online motion compensation system for scanned particle beams. Phys Med Biol 2006;51:3517-31.

[16] Lujan AE, Larsen EW, Balter JM, Haken RKT. A method for incorporating organ motion due to breathing into 3D dose calculations. Med Phys 1999;26:715-20.

[17] Chaudhri N, Saito N, Bert C, Franczak B, Steidl P, Durante M, et al. Ion-optical studies for a range adaptation method in ion beam therapy using a static wedge degrader combined with magnetic beam deflection. Phys Med Biol 2010;55:3499-513. 